\title{
Erratum to: Aging Locality Awareness in Cost Estimation for Database Query Optimization
}

\author{
Chihiro Kato ${ }^{1(\bowtie)}$, Yuto Hayamizu ${ }^{1}$, Kazuo Goda ${ }^{1}$, \\ and Masaru Kitsuregawa ${ }^{1,2}$ \\ 1 The University of Tokyo, Komaba 4-6-1, Meguro-ku, Tokyo, Japan \\ kato@tkl.iis.u-tokyo.ac.jp \\ 2 National Institute of Informatics, Hitotsubashi 2-1-2, Chiyoda-ku, Tokyo, Japan \\ http: //www. u-tokyo.ac.jp/ \\ http://www.nii.ac.jp/
}

\section{Erratum to: \\ Chapter 32: S. Hartmann and H. Ma (Eds.) \\ Database and Expert Systems Applications \\ DOI: 10.1007/978-3-319-44406-2_32}

In an older version of the paper starting on p. 389 of the DEXA proceedings (LNCS 9828), the authors' affiliations were incorrect. This has been corrected. 